\title{
Prospective estimation of mean axon diameter and extra-axonal space of the posterior limb of the internal capsule in patients with idiopathic normal pressure hydrocephalus before and after a lumboperitoneal shunt by using q-space diffusion MRI
}

\author{
Masaaki Hori $^{1}$ (D) Kouhei Kamiya $^{1,2} \cdot$ Atsushi Nakanishi $^{1} \cdot$ Issei Fukunaga $^{1,3}$. \\ Masakazu Miyajima ${ }^{4}$ Madoka Nakajima $^{4} \cdot$ Michimasa Suzuki $^{1} \cdot$ Yuriko Suzuki $^{1,5}$. \\ Ryusuke Irie $^{1,2} \cdot$ Koji Kamagata $^{1} \cdot$ Hajime Arai $^{4} \cdot$ Shigeki Aoki $^{1}$
}

Received: 29 September 2015 /Revised: 20 November 2015 / Accepted: 7 December 2015 / Published online: 22 December 2015

(C) The Author(s) 2015. This article is published with open access at Springerlink.com

\begin{abstract}
Objectives To prospectively estimate the mean axon diameter (MAD) and extracellular space of the posterior limb of the internal capsule (PLIC) in patients with idiopathic normal pressure hydrocephalus (iNPH) before and after a lumboperitoneal (LP) shunting operation using q-space diffusion MRI analysis.

Methods We studied 12 consecutive patients with iNPH and 12 controls at our institution. After conventional magnetic resonance imaging (MRI), q-space image (QSI) data were acquired with a 3-T MRI scanner. The MAD and extraaxonal space of the PLIC before and after LP shunting were calculated using two-component q-space imaging analyses; the before and after values were compared.

Results After LP shunt surgery, the extracellular space of the PLIC was significantly higher than that of the same patients before the operation (one-way analysis of
\end{abstract}

Masaaki Hori

mahori@juntendo.ac.jp

1 Department of Radiology, Juntendo University School of Medicine, 2-1-1 Hongo, Bunkyo-ku, Tokyo 113-8421, Japan

2 Department of Radiology, The University of Tokyo, 7-3-1 Hongo, Bunkyo-ku, Tokyo 113-8655, Japan

3 Department of Health Science, Graduate School of Human Health Sciences, Tokyo Metropolitan University, 7-2-10, Higashiogu, Arakawa-ku, Tokyo 116-8551, Japan

4 Department of Neurosurgery, Juntendo University School of Medicine, 2-1-1 Hongo, Bunkyo-ku, Tokyo 113-8421, Japan

5 C.J. Gorter Center for High Field MRI, Department of Radiology, Leiden University Medical Center, Leiden 2333 ZA, Netherlands variance (ANOVA) with Scheffé's post-hoc test, $P=0.024)$. No significant differences were observed in the PLIC axon diameters among normal controls or in patients before and after surgery.

Conclusion Increases in the root mean square displacement in the extra-axonal space of the PLIC in patients with iNPH after an LP shunt procedure are associated with the microstructural changes of white matter and subsequent abatement of patient symptoms.

Key Points

- Q-space diffusion MRI provides information on microstructural changes in the corticospinal tract

- Lumboperitoneal (LP) shunting operation is useful for idiopathic normal pressure hydrocephalus

- Q-space measurement may be a biomarker for the effect of the LP shunt procedure

Keywords Idiopathic NPH · Lumboperitoneal shunt · Q-space imaging · Axon diameter · Diffusion MRI

$\begin{array}{ll}\text { Abbreviations } \\ \text { ADC } & \text { Apparent diffusion coefficient } \\ \text { ANOVA } & \text { One way analysis of variance } \\ \text { CST } & \text { Corticospinal white matter tract } \\ \text { DKI } & \text { Diffusion kurtosis imaging } \\ \text { DTI } & \text { Diffusion tensor imaging } \\ \text { FA } & \text { Fractional anisotropy } \\ \text { ICC } & \text { Intraclass correlation coefficient } \\ \text { iNPH } & \text { Idiopathic normal pressure hydrocephalus } \\ \text { LP } & \text { Lumboperitoneal } \\ \text { MAD } & \text { Mean axon diameter } \\ \text { PLIC } & \text { Posterior limb of the internal capsule }\end{array}$


QSI Q-space imaging

RMSD Root mean square displacement

ROI Region(s) of interest

\section{Introduction}

Idiopathic normal pressure hydrocephalus (iNPH) is a disease of unknown aetiology. Its major triad of symptoms consists of gait disturbance, dementia and bladder incontinence. Unlike other neurodegenerative diseases, iNPH can be relieved by a neurosurgical operation to drain excess cerebrospinal fluid (CSF). In fact, decreased gait disturbance is seen in patients with iNPH after shunt surgery; however, the mechanism of clinical improvement after surgery, as well as the origin of gait disturbance in iNPH, remains unknown. Several hypotheses have been reported, such as frontal lobe dysfunction [1] and/or malfunction of the cortex-basal gangliathalamus-cortex circuit, including the supplementary motor cortex [2]. Other pathological changes observed in the brains of patients with iNPH, such as ischaemia and gliosis due to transependymal diapedesis of the CSF, may be related to gait disturbance [3]. One possible explanation is the compression or deformation of the corticospinal white matter tract (CST) - a hypothesis supported by recent diffusion tensor imaging (DTI) and diffusion kurtosis imaging (DKI) studies [4, 5]. In this view, the decreased gait disturbance after shunt surgery is explained in part by partial decompression of the white matter tracts and their return to their original shapes and positions.

Recently, several methods of measuring the axonal diameter or density by diffusion magnetic resonance imaging (MRI) have been proposed to investigate microstructure and its changes in the brain, especially in axons [6-10]. These methods usually require high-performance MRI hardware with features such as high gradient amplitudes and long scanning times, which are beyond the tolerable limits for clinical application. However, one of these techniques - the twocomponent low-q fit method - was introduced as a way to measure the axonal diameter by using q-space diffusion MRI with fewer demands in terms of hardware and scanning time. In this method, the q-space echo attenuation comprises both extra- or intracellular space (ECS and ICS, respectively) signals in white matter; the echo attenuations are fit to a twocompartment model. Ong et al. [11, 12] first quantified mean axon diameter (MAD) calculated from ICS in excised mouse spinal cord tissue with a 9.4-T MR imager, and later showed the feasibility of applying this technique to a 1.5-T MR imager [13], albeit with a scanning time of up to $5 \mathrm{~h}$. Although the scan duration must be shortened to be clinically tolerable, we expected that the method described above would provide valuable information on structural and pathological changes in the white matter tract in vivo. In fact, Suzuki et al. [14] reported the differences of axon diameter at each area of normal corpus callosum in vivo, and Kamiya et al. [15] reported significant increases in the CST axon density at the level of the ventricular body in patients with iNPH, compared with normal volunteers using this technique with a 3-T clinical MR scanner [15]. It may be reasonable to perform measurements of QSI indices at the periventricular area; however, the microstructural changes of CST distant from the area remain to be understood.

Therefore, the aim in this study was to prospectively estimate the MAD and extra-axonal space of the posterior limb of the internal capsule (PLIC) in patients with iNPH before and after a lumboperitoneal (LP) shunting operation using q-space diffusion analysis.

\section{Materials and methods}

\section{Subjects}

Between 11 December 2010 and 23 June 2014 a total of 24 consecutive patients with strongly suspected iNPH from other hospitals who had planned to undergo LP shunt surgery were referred to our hospital and participated in this prospective study. However, QSI data were not obtained in 12 patients after the surgical procedure because follow-ups were conducted at other hospitals. Therefore, only 12 patients out of 24 were included in this study. Patients referred to our hospital previously underwent conventional MRI including T2-, T2*-, T1- and diffusion-weighted imaging at other hospitals because of the social insurance system in our country.

Diagnosis of iNPH was made based on the typical clinical symptoms (i.e. gait disturbance, cognitive impairment and urinary incontinence) and ventricular dilatation as confirmed by conventional MRI according to the guidelines for management of iNPH, second edition, as clinical diagnostic criteria of possible iNPH with MRI support [16]. Possible iNPH requires all of the five following criteria: (a) developing symptoms at an age $>60$ years; (b) more than one of the three clinical symptoms mentioned above; (c) ventricular dilatation (Evans index $>0.3$ ); (d) the clinical symptoms cannot be explained by any other disease; and (e) preceding disease possibly causing ventricular dilatation, such as subarachnoid haemorrhage, is not obvious. All patients were evaluated using the 3-m timed up-and-go test (TUG) for gait disturbance, the Mini-Mental State Examination (MMSE) for cognitive impairment, and self-reported urinary incontinence diagnosed by two experienced neurosurgeons. Their demographic characteristics are summarized in Table 1. Moreover, MRI diagnosis in the guidelines requires that MRI shows narrowing of the sulci and subarachnoid space over the high convexity/midline surface.

Exclusion criteria were as follows: (a) the presence of additional intraspinal disease (e.g. a tumour or inflammatory 
Table 1 Demographic characteristics of the subjects

\begin{tabular}{llll}
\hline & Control & iNPH (before surgery) & iNPH (after surgery) \\
\hline N (female/male) & $12(5 / 7)$ & $12(6 / 6)$ & \\
Age (years) & $75.0 \pm 1.3$ & $75.0 \pm 1.3$ & \\
TUG, time to complete (sec) & NA & $19.0 \pm 1.0$ & $13.5 \pm 2.2$ \\
TUG, number of steps & NA & $23.1 \pm 4.6$ & $15.2 \pm 1.8$ \\
MMSE & NA & $23.8 \pm 1.3$ & $26.6 \pm 0.7$ \\
Urinary incontinence & NA & 5 & 1 \\
Evans index & NA & $0.348 \pm 0.008$ & $0.341 \pm 0.101$ \\
\hline
\end{tabular}

Values for age, TUG, MMSE and Evans index are given as mean \pm standard error

TUG Timed Up-and-Go Test, MMSE Mini-Mental State Examination, iNPH idiopathic normal pressure hydrocephalus disease); (b) the presence of another neurological disease (e.g. a neoplasm) in the brain upon conventional MRI (T1- or T2weighted); (c) a history of neurological disease; (d) a history of head surgery for any disease; and (e) unsatisfactory image quality for analysis. In addition, 12 age-matched normal control subjects were included for reference. They were recruited from our hospital and had minimal neurological symptoms (i.e. headache) and no abnormalities on screening with conventional MRIs, as confirmed by neuroradiologists.

Informed consent was obtained from all patients and control subjects. We obtained ethical approval from the institutional review board before the study. The median intervals from the date of the first MRI to the LP shunt surgery and from the surgery to the second MRI scan were $33 \pm 14$ days (median \pm standard error) and $97.5 \pm 32$ days, respectively.

\section{Image acquisition}

Patients underwent MR examination on a 3-T MR scanner (Achieva; Philips Medical Systems, Best, The Netherlands) equipped with gradient hardware allowing up to $80 \mathrm{mT} / \mathrm{m}$ before and after LP shunt surgery. Data were acquired using an MRI protocol that included axial fluid-attenuated inversion recovery (FLAIR), three-dimensional (3D) T1-weighted and q-space imaging using single-shot echo-planar sequence. FLAIR images were obtained with fast spin-echo imaging: repetition time (ms)/echo time (ms), 12,000/120; number of excitations, two; section thickness/gap, $5 / 1 \mathrm{~mm}$; number of sections, 22; field of view (FOV), $230 \times 230 \mathrm{~mm}$; matrix, $512 \times 512$; flip angle, $90^{\circ}$. 3D T1-weighted sagittal images were obtained with 3D fast-field echo imaging: repetition time (ms)/echo time (ms), 15/3.54; number of excitations, one; section thickness/gap, 0.86/0 mm; number of sections, 190; FOV, $260 \times 260 \mathrm{~mm}$; matrix, $320 \times 320$; flip angle, $10^{\circ}$.

The parameters used for QSI were as follows: repetition time (ms)/echo time (ms), 8,000/96; number of excitations, one; section thickness/gap, 4/0 mm; number of sections, 20; FOV, $256 \times 256 \mathrm{~mm}$; matrix, $64 \times 64$; imaging time, approximately $7 \mathrm{~min}$; and $16 \mathrm{~b}$-values $(0,124,496,1,116,1,983,3$,
099, 4,463, 6,074, 7,934, 10,041, 12,397 and 15,000 s/mm with diffusion encoding in one direction, $\mathrm{x}, \mathrm{y}, \mathrm{z}=0.7071$, $-0.7071,0)$ for each $b$-value. The gradient length $(\delta)$ and time between the two leading edges of the diffusion gradient $(\Delta)$ were 37.8 and $47.3 \mathrm{~ms}$, respectively. The reciprocal space vector $\mathrm{q}$ is defined by the following equation:

$\mathrm{q}=\gamma \mathrm{G} \delta / 2 \pi$

where $\gamma$ is the gyromagnetic ratio and $\mathrm{G}$ is the amplitude of the diffusion gradients.

Therefore, the corresponding q-values for $b$-values were 0 , 95.1, 190.3, 285.4, 380.5, 475.6, 570.8, 665.9, 761.0, $856.1,951.3$ and $1,046.4 \mathrm{~cm}^{-1}$ (Fig. 1). The q-value 1, $046.4 \mathrm{~cm}^{-1}$ is the highest q-value for our MR scanner in this condition.

\section{Two-component q-space analysis of posterior limb of the internal capsule (PLIC)}

Two-component QSI analyses were performed by using an m-file program custom-developed with MATLAB software (The MathWorks Inc., Natick, MA, USA) on an independent Windows PC. This analysis necessitates a diffusion gradient perpendicular to the CST. The known CST course is usually perpendicular to the axial imaging plane [17]. Therefore, we applied a diffusion gradient perpendicular to the superiorinferior axis; the CST could be identified as a hyperintense tract running from the precentral gyrus to the cerebral peduncle through the PLIC [18]. Regions of interest (ROIs) were placed manually on the bilateral PLICs at the level of the internal capsule (Fig. 2A) by two observers (F.I., 5 years of experience in MR research, and M.H., 16 years of experience in neuroradiology) independently without knowledge of clinical findings. The ROIs included the bright appearing CST on $\mathrm{q}=380.5$ and $475.6 \mathrm{~cm}^{-1}$ images. The cranial and caudal images and different q-value images were also used as references. As described in previous papers, the root mean square displacements (RMSDs) of intra-axonal space, 
Fig. 1 Examples of the diffusionweighted images at different qvalues (A) and T1-weighted images in the axial and coronal planes $(\mathbf{B})$ in a patient with idiopathic normal pressure hydrocephalus (iNPH)
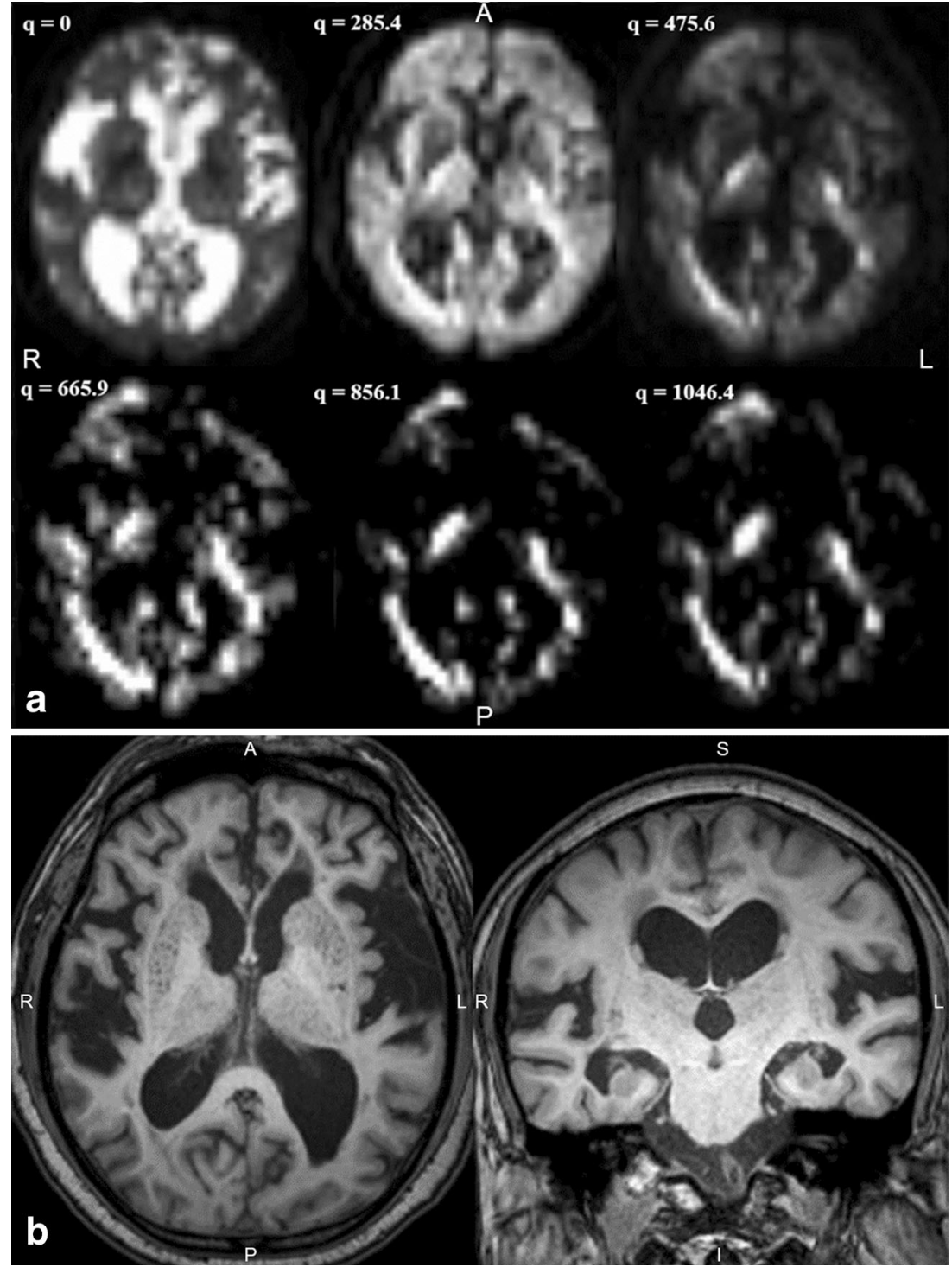

extra-axonal space and intra-axonal volume fraction of the PLICs can be determined by acquiring data at multiple $q$ values (including $\mathrm{q}=0$ ) and by fitting them to the following equation [9]:

$$
\begin{aligned}
E(q)= & \left(1-f_{I C S}\right) \exp \left(-2 \pi^{2} q^{2} Z_{E C S}{ }^{2}\right) \\
& +f_{I C S} \exp \left(-2 \pi^{2} q^{2} Z_{I C S}{ }^{2}\right)
\end{aligned}
$$

where $E(q)$ is the q-space signal decay, $f_{I C S}$ is the relaxationweighted intracellular volume fraction, and $\mathrm{Z}_{\mathrm{ECS}}$ and $\mathrm{Z}_{\mathrm{ICS}}$ are the RMSDs of diffusing molecules in the extra- and intracellular spaces, respectively (Fig. 2B). We hypothesized that the RMSD of the intra-axonal space reflects the mean axonal diameter (MAD) of the PLICs based on the theory of low q-value method [9]. The coefficient of determination $\left(\mathrm{R}^{2}\right)$ of the fitted curve for each ROI was also recorded.

\section{Statistical analysis}

Statistical analyses were performed using SPSS Statistics software (version 19.0; IBM, Chicago, IL, USA). We tested the side differences in the RMSD values and intracellular volume fractions in healthy control subjects and iNPH patients by using a paired-sample t-test, and we found no significant differences $(\mathrm{P}>.05)$. Therefore, the RMSD values in the extraand intracellular spaces and intracellular volume fraction were averaged bilaterally for statistical analysis. Moreover, interobserver variability for the RMSD ROI measurements of the two readers was analysed by calculating the intraclass correlation coefficient (ICC; $0.00-0.20$ poor, $0.21-0.40$ fair, $0.41-0.60$ moderate, $0.61-0.80$ good and $0.81-1.00$ excellent correlation). RMSD values and intracellular volume fractions were averaged between the two observers for further analyses. Statistical comparisons using one-way ANOVA with Scheffé's 
post-hoc test among the values of normal controls, before and after LP shunting, were performed. The significance criterion was set to $P<0.05$ to indicate a statistically significant difference.

\section{Results}

Twelve patients were successfully scanned before and after the LP shunt operation and included in the evaluation. No patient was excluded from analysis because of image degeneration.

Excellent fits were obtained for all ROIs $\left(\mathrm{R}^{2}>0.95\right)$. RMSD values ( $\mu \mathrm{m}$, mean \pm standard error) of extra- and intracellular spaces and intracellular volume fraction in the PLIC of the 12 normal controls and 12 patients with iNPH are summarized in Table 2. After surgery, the RMSD value of extracellular spaces in the PLIC of the same 12 patients was significantly higher than that of those patients before the operation (one-way analysis of variance (ANOVA) with Scheffe's post-hoc test, $P=0.013$ ). No significant difference was observed in the MAD and intra-axonal volume fraction in the PLIC among normal controls or patients before and after surgery. We confirmed that inter-observer reproducibility was excellent (ICC 0.91) for the RMSD measurements of extracellular spaces (ICC 0.92), MAD (ICC 0.90) and intracellular volume fraction (ICC 0.88).

Alleviation of clinical symptoms, including gait disturbance by TUG, cognitive impairment by MMSE and urinary incontinence by self-assessment were confirmed by the same two neurosurgeons in the 12 patients who underwent MR examinations after the LP shunt surgery. Their demographic characteristics are summarized in Table 1 . In all patients, clinical symptoms were improved after LP shunts, and there were no statistical correlations between RSMD values and clinical scores.

\section{Discussion}

Our findings of unchanged MAD and intra-axonal volume fraction in the PLIC of normal controls and iNPH patients are consistent with those of a previous study [15]. Moreover, the higher RMSD values for extracellular space and unchanged MAD in the PLIC after LP shunting suggest that the axons of the CSTs in patients with iNPH were not fatally damaged. In other words, the extra-axonal water component may have decreased reversibly, and the axons themselves may be deformed but not destroyed when the CSTs were compressed by the dilated ventricles. This could in part explain why the clinical symptoms were relieved after appropriate shunt surgery.
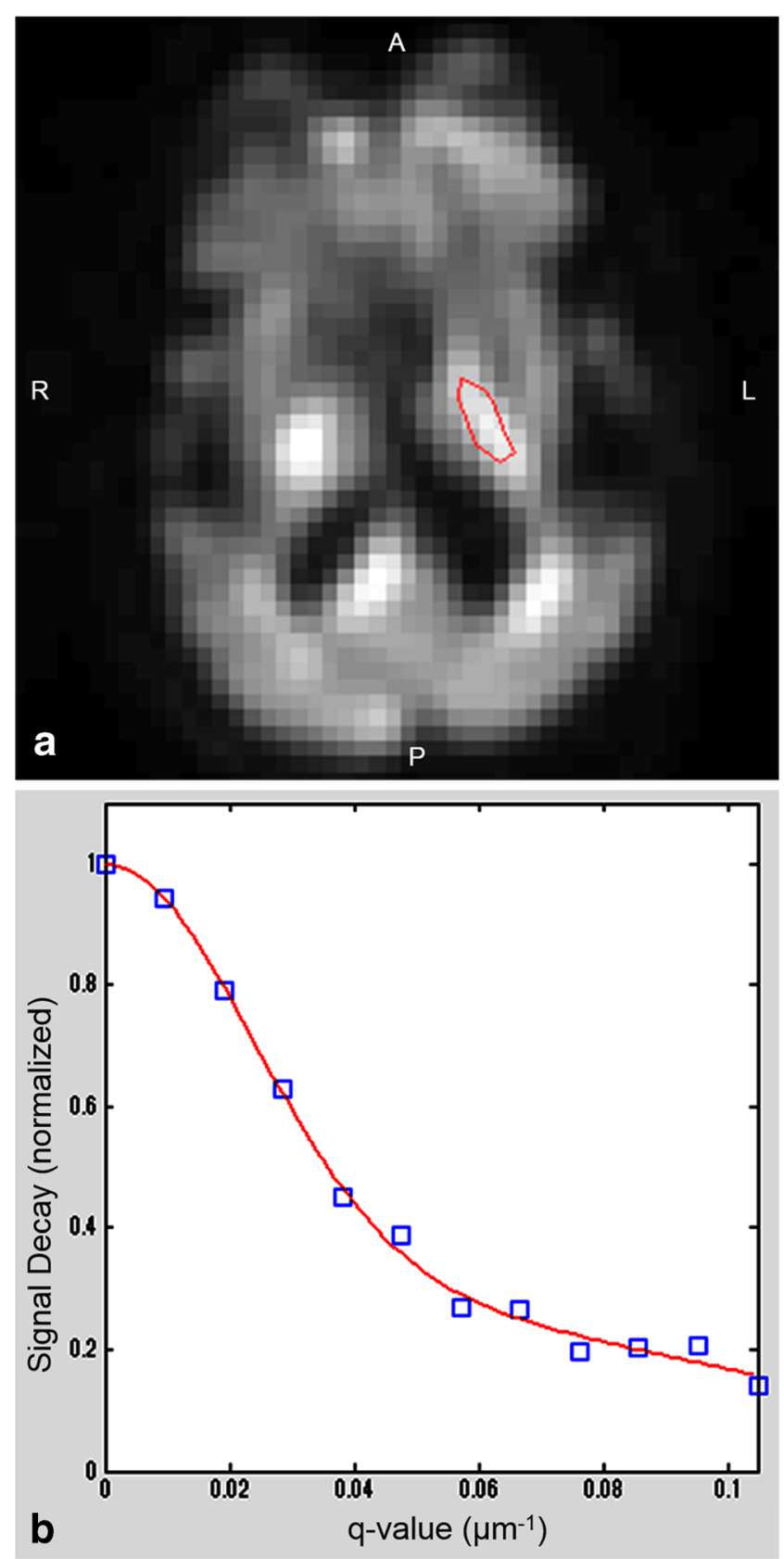

Fig. 2 A representative diffusion-weighted image with a region of interest placed on the left posterior limb of the internal capsule (PLIC; A) and an example of q-space signal decay for the left PLIC and fitted two-component curve in a patient with Idiopathic normal pressure hydrocephalus (iNPH) before the lumboperitoneal (LP) shunting surgical operation (B). Note that the two-component computed fit for signal decay curve was performed for averaged pixel values in the region of interest

In addition to these findings, the postoperative extra-axonal space seems to be increased compared with that in normal controls. We speculate that the increased extra-axonal space (i.e. interstitial space) is the compensatory change for the decreased CSF volume occurring forcefully by the LP shunt procedure. If the extra-axonal space is restored to normal 
Table 2 Root mean square displacement (RMSD) values of extra- and intracellular spaces and intracellular volume fraction in the posterior limb of the internal capsule of normal controls and patients with idiopathic normal pressure hydrocephalus (iNPH)

\begin{tabular}{llll}
\hline & $\begin{array}{l}\text { Control } \\
(\mathrm{N}=12)\end{array}$ & $\begin{array}{l}\text { iNPH (before surgery) } \\
(\mathrm{N}=12)\end{array}$ & $\begin{array}{l}\text { iNPH (after surgery) } \\
(\mathrm{N}=12)\end{array}$ \\
\hline Axon diameter $(\mu \mathrm{m})$ & $2.03 \pm 0.11$ & $2.00 \pm 0.12$ & $2.19 \pm 0.09$ \\
RMSD of extra-axonal space $(\mu \mathrm{m})$ & $8.04 \pm 0.28$ & $7.67 \pm 0.30^{*}$ & $8.96 \pm 0.31^{*}$ \\
Intra-axonal volume fraction & $0.40 \pm 0.02$ & $0.36 \pm 0.01$ & $0.38 \pm 0.02$ \\
\hline
\end{tabular}

Values are given as mean \pm standard error

*Significant difference (ANOVA with Scheff's post-hoc test, $P=0.013$ ) levels as reasonably expected, the ventricles will expand to the pre-operation level. To suppress this phenomenon, extraaxonal space may increase in patients with iNPH after the LP shunt compared with that in normal subjects.

With regard to PLIC assessment in patients with iNPH by diffusion metrics, increased fractional anisotropy (FA) and apparent diffusion coefficient (ADC), as well as decreased mean kurtosis, has been reported in the literature [4]. Although these results support the compaction and stretching model of compressed white matter fibres in the CST, they do not help explain the clinically reversible symptoms. Recently, a QSI with larger and more numerous b-values has been reported as an advanced diffusion MRI technique in clinical settings [19]. Some reports have shown QSIs to have promising advantages over conventional diffusion metrics, for example in patients with multiple sclerosis [20-23] and cerebral infarction [24]. However, the RMSD value of one voxel is the mean RMSD of various components, despite using the QSI technique, because one voxel of neural tissue is usually more than $2 \mathrm{~mm}^{3}$ in clinical settings and consists of many components (e.g. axons) with various RMSD values. Therefore, there is a report showing the limitations of usefulness of RMSD derived from a QSI [25]. In contrast, we calculated the RMSD values by separating the tissue into two components (i.e., intra- and extra-axonal) using multiple bvalues. Therefore, our results may reveal more detailed microstructural changes in the neural tissue.

One potential limitation of the current study is the limited direction and number of motion probe gradient pulses that can be used to measure only the intra- and extra-axonal spaces of corticospinal tracts. This is because the method used here is appropriate only under the limited condition that white matter fibres run perpendicular to the imaging plane. With more directions and $b$-values, other white matter tracts can also be measured, and direct comparisons can be made between the RMSD values of extracellular spaces, MAD values and diffusion metrics (e.g. FA and ADC). Currently, the scanning time for a two-component QSI is $7 \mathrm{~min}$ in clinical settings; more directions and $b$-values will thus increase the examination time multiplicatively - a circumstance that is beyond tolerable limits for patients. This problem will likely be resolved in future studies by using imaging techniques such as multiband echo-planar imaging [26]. Moreover, low spatial resolution of the QSI in this study also limits the quality and accuracy of the analysis. This problem is due to the examination time and hardware of the MRI system.

Another limitation of the current study is that the determination of diffusion gradient direction used an approximate method; therefore, it could have been different from the running course perpendicular to the CST. In a past report, CST tractography in patients with iNPH seemed to have minor changes in proper diffusion gradient orientation [4]. However, we cannot guarantee the same diffusion weighting gradient orientation between the subjects or scan sessions in our study. One potential solution is that the appropriate direction of the diffusion gradient is determined by CST tractography based on diffusion tensor MR data and prepared before QSI data acquisition, but this seems to be difficult for clinical examinations.

The other limitation is that motion correction or coregistration between the images at different $b$-values and diffusion gradient orientations was not applied in our study. We have applied these techniques to data, but they did not work well because of the markedly different shapes and contrasts of the high $b$-value diffusion MRI data. One technique has been proposed using experimental DTI data and simulations using the composite hindered and restricted model of diffusion framework for correction [27], but DTI analysis was not able to be applied to our data because our data consist of only one MPG diffusion direction. The methods for high $b$-value dMRI remain to be established.

Moreover, another important technical limitation is that the short gradient pulse (SGP) approximation $(\delta<<$ ) was not fulfilled in our QSI because of the limited maximum magnetic field gradient strength in a clinical 3-T MRI system. A possible drawback of the unsatisfactory SGP is that it results in an underestimation of RMSD in q-space analysis [28]. Therefore, values for MAD in our methods should be treated as apparent values and used for monitoring changes of white matter, i.e. intergroup comparison.

Another limitation is the small number of patients for whom pre- and postoperative data were obtained and in whom gait disturbances decreased after the LP shunt procedure. Future studies with larger numbers of patients are needed to establish the usefulness of MAD measurements in the PLIC of patients with iNPH.

In conclusion, increases in RMSD in the extra-axonal space of the PLIC according to q-space diffusion analysis in 
patients with iNPH after a LP shunt procedure are associated with microstructural changes of white matter and subsequent abatement of patient symptoms. The proposed QSI technique has the potential to provide novel information beyond that provided by conventional MRI. Moreover, a QSI may provide useful information on in vivo biomarkers for future studies, particularly in relation to patient prognosis.

Acknowledgements The scientific guarantor of this publication is Prof. Shigeki Aoki, Department of Radiology, Juntendo University School of Medicine. The authors of this manuscript declare relationships with the following company: Philips Electronics Japan, Ltd. One of the co-authors was an employee of Philips Electronics Japan, Ltd. This study received funding in the form of partial support from a Research Grant from the Ministry of Health, Labor and Welfare of Japan (2011-Nanchi-018) and a Grant-in-Aid for Scientific Research on Innovative Areas (Comprehensive Brain Science Network) from the Ministry of Education, Culture, Sports, Science and Technology of Japan (221S0003). No complex statistical methods were necessary for this paper. Institutional Review Board approval was obtained. Written informed consent was obtained from all subjects (patients) in this study. Study subjects or cohorts have not been previously reported. Methodology: prospective, case-control study, performed at one institution.

Open Access This article is distributed under the terms of the Creative Commons Attribution-NonCommercial 4.0 International License (http:// creativecommons.org/licenses/by-nc/4.0/), which permits any noncommercial use, distribution, and reproduction in any medium, provided you give appropriate credit to the original author(s) and the source, provide a link to the Creative Commons license, and indicate if changes were made.

\section{References}

1. Stolze H, Kuhtz-Buschbeck JP, Drücke H, Jöhnk K, Illert M, Deuschl G (2001) Comparative analysis of the gait disorder of normal pressure hydrocephalus and Parkinson's disease. J Neurol Neurosurg Psychiatry 70:289-297

2. Akai K, Uchigasaki S, Tanaka U, Komatsu A (1987) Normal pressure hydrocephalus. Neuropathological study. Acta Pathol Jpn 37: 97-110

3. Krauss JK, Regel JP, Vach W, Droste DW, Borremans JJ, Mergner T (1996) Vascular risk factors and arteriosclerotic disease in idiopathic normal-pressure hydrocephalus of the elderly. Stroke 27:24-29

4. Nakanishi A, Fukunaga I, Hori M et al (2013) Microstructural changes of the corticospinal tract in idiopathic normal pressure hydrocephalus: a comparison of diffusion tensor and diffusional kurtosis imaging. Neuroradiology 55:971-976

5. Hattori T, Ito K, Aoki S et al (2013) White matter alteration in idiopathic normal pressure hydrocephalus: tract-based spatial statistics study. AJNR Am J Neuroradiol 33:97-103

6. Assaf Y, Blumenfeld-Katzir T, Yovel Y, Basser PJ (2008) AxCaliber: a method for measuring axon diameter distribution from diffusion MRI. Magn Reson Med 59:1347-1354

7. Alexander DC, Hubbard PL, Hall MG et al (2010) Orientationally invariant indices of axon diameter and density from diffusion MRI. Neuroimage 52:1374-89

8. Assaf Y, Alexander DC, Jones DK et al (2013) The CONNECT project: combining macro- and micro-structure. Neuroimage 80 : 273-282

9. McNab JA, Edlow BL, Witzel T et al (2013) The Human Connectome Project and beyond: initial applications of $300 \mathrm{mT} / \mathrm{m}$ gradients. Neuroimage 80:234-245
10. Barazany D, Basser PJ, Assaf Y (2009) In vivo measurement of axon diameter distribution in the corpus callosum of rat brain. Brain 132:1210-1220

11. Ong HH, Wright AC, Wehrli SL et al (2008) Indirect measurement of regional axon diameter in excised mouse spinal cord with q-space imaging: simulation and experimental studies. Neuroimage 40:1619-1632

12. Ong HH, Wehrli FW (2010) Quantifying axon diameter and intracellular volume fraction in excised mouse spinal cord with q-space imaging. Neuroimage 51:1360-1366

13. Ong HH, Bhagat Y, Magland J, Wehrli FW (2012) Feasibility of low q-space diffusion MRI at 1 5T. ISMRM 20th Annual Meeting \& Exhibition Melbourne, Victoria, Australia

14. Suzuki Y, Hori M, Kamiya K, Fukunaga I, Aoki S, VAN Cauteren M (2015) Estimation of the mean axon diameter and intra-axonal space volume fraction of the human corpus callosum: diffusion qspace imaging with low q-values. Magn Reson Med Sci. doi:10. 2463/mrms.2014-0141

15. Kamiya K, Hori M, Miyajima M et al (2014) Axon diameter and intra-axonal volume fraction of the corticospinal tract in idiopathic normal pressure hydrocephalus measured by q-space imaging. PLoS One 9, e103842

16. Mori E, Ishikawa M, Kato T et al (2012) Guidelines for management of idiopathic normal pressure hydrocephalus: second edition. Neurol Med Chir (Tokyo) 52:775-809

17. Yamada K, Kizu O, Kubota T et al (2007) The pyramidal tract has a predictable course through the centrum semiovale: a diffusion-tensor based tractography study. J Magn Reson Imaging 26:519-524

18. Schaefer PW, Grant PE, Gonzalez RG (2000) Diffusion-weighted MR imaging of the brain. Radiology 217:331-345

19. Hori M, Fukunaga I, Masutani Y et al (2012) Visualizing nonGaussian diffusion: clinical application of q-space imaging and diffusional kurtosis imaging of the brain and spine. Magn Reson Med Sci 11:221-333

20. Assaf Y, Chapman J, Ben-Bashat D et al (2005) White matter changes in multiple sclerosis: correlation of q-space diffusion MRI and $1 \mathrm{H}$ MRS. Magn Reson Imaging 23:703-710

21. Fatima Z, Motosugi U, Hori M et al (2012) High b-value q-space analyzed diffusion-weighted MRI using 15 Tesla clinical scanner: determination of displacement parameters in the brains of normal versus multiple sclerosis and low-grade glioma subjects. J Neuroimaging 22:279-284

22. Hori M, Yoshida M, Yokoyama K et al (2014) Multiple sclerosis: benefits of q-space imaging in evaluation of normal-appearing and periplaque white matter. Magn Reson Imaging 32:625-629

23. Farrell JA, Smith SA, Gordon-Lipkin EM, Reich DS, Calabresi PA, van Zijl PC (2008) High b-value q-space diffusion-weighted MRI of the human cervical spinal cord in vivo: feasibility and application to multiple sclerosis. Magn Reson Med 59:1079-1089

24. Hori M, Motosugi U, Fatima Z et al (2011) A comparison of mean displacement values using high b-value q-space diffusion-weighted MRI with conventional apparent diffusion coefficients in patients with stroke. Acad Radiol 18:837-841

25. Fatima Z, Motosugi U, Waqar AB et al (2013) Associations among q-space MRI, diffusion-weighted MRI and histopathological parameters in meningiomas. Eur Radiol 23:2258-2263

26. Xu J, Moeller S, Auerbach EJ et al (2013) Evaluation of slice accelerations using multiband echo planar imaging at $3 \mathrm{~T}$. Neuroimage 83:991-1001

27. Ben-Amitay S, Jones DK, Assaf Y (2012) Motion correction and registration of high b-value diffusion weighted images. Magn Reson Med 67:1694-1702

28. Bar-Shir A, Avram L, Ozarslan E, Basser PJ, Cohen Y (2008) The effect of the diffusion time and pulse gradient duration ratio on the diffraction pattern and the structural information estimated from qspace diffusion MR: experiments and simulations. J Magn Reson 194:230-236 